\title{
Cesarean scar defect- obstetric consequences
}

\author{
Oana D. Balalau ${ }^{1,2}$, Teodora A. Corbu ${ }^{2}$, Liana Ples ${ }^{1,2}$, Vasile A. Dumitru ${ }^{1,3}$, Anca D. \\ Stanescu $^{1,2}$
}

${ }^{1}$ Carol Davila University of Medicine and Pharmacy, Bucharest, Romania

${ }^{2}$ St. John Emergency Hospital, Department of Bucur Maternity, Bucharest, Romania

${ }^{3}$ Emergency University Hospital Bucharest, Department of Pathology, Bucharest, Romania

\begin{abstract}
Scope. This review aims to achieve a parallelism between literature studies on imaging diagnosis, the characteristics of cesarean scar defect and its evolution during pregnancy, and the obstetric consequences that can lead to increased maternal morbidity.

Materials and Methods. There have been many literature studies published in recent years aimed to identify the methods of diagnosis for cesarean scar defect, the characteristics and the main changes of it during pregnancy, and the risks undertaken by pregnant women undergoing labor for vaginal delivery after a previous cesarean surgery.

Results and Discussions. The most commonly used method for visualizing and evaluating uterine scar is transvaginal ultrasound in the first trimester of pregnancy, and abdominal ultrasonography in the II and III trimesters. The two areas characteristic of uterine scarring: "niche" and "thickness of the residual myometrium" undergo changes during the three trimesters of pregnancy. Some studies in the literature reported a higher incidence of uterine rupture, especially when the lower uterine segment fell below $2.3 \mathrm{~mm}$, and a mean thickness of $3.2 \mathrm{~mm}$, higher among multiparous women. A question that still does not have a generally valid answer refers to the hysterorrhaphy technique. There are different opinions among the authors, but most of them consider that after a double layer suture the thickness of the residual myometrium is higher. However, data from the literature show that the incidence of complications such as uterine rupture, dehiscence of the uterine trance are similar, regardless of the type of suture used. Another complication that occurs due to uterine scar is the pregnancy inserted at this level, which in the case of expectancy, leads to an increase in maternal and fetal morbidity. $50 \%$ of them evolve to spontaneous abortion in the first trimester, and almost all full-term pregnancies result in hysterectomy of necessity.

Conclusions. Due to the many obstetrical and gynecological complications that occur on a uterine scar, an evaluation in dynamics is essential. There are no standardized protocols yet, but the identification and measurement of the uterine scar area in the third trimester may predict the chance of success of the labor test for vaginal delivery after cesarean surgery.
\end{abstract}

\section{Keywords $\quad$ : uterine scar, cesarean section, vaginal birth}

Highlights

$\checkmark$ The success of the labor test for vaginal delivery after cesarean surgery is difficult to predict, but it seems that the characteristics of uterine scar have an important role.

$\checkmark$ Complications that may occur in the presence of uterine scar lead to increased maternal and fetal morbidity, making it an important topic to be debate.

To cite this article: Balalau DO, Corbu TA, Ples L, Dumitru VA, Stanescu AD. Cesarean scar defect- obstetric consequences. J Clin Invest Surg. 2018; 3(2): 72-76. DOI: $10.25083 / 2559.5555 / 3.2 / 72.76$ 


\section{Introduction}

Uterine scar is one of the main determinants of the prognosis for a pregnancy following previous caesarean section delivery. Interest in evaluating the uterine scar has grown in recent years, as evidenced by the multitude of international studies published in the literature, regarding the evolution of the uterine scar, the healing process, as well as the consequences for both nonpregnant and pregnant women. The rate of cesarean operation is steadily increasing (reaching $30 \%$ in the US and $25 \%$ in Europe), and the number of pregnant women with a history of caesarean section also $(1,2)$, therefore the need to highlight the methods of predicting birth success naturally, following previous cesarean delivery. Imagistic evaluation of uterine scar is currently enjoying a wide range of techniques to identify, locate and measure the three dimensions of the defect at this level as well as the thickness of the residual myometrium, that can be done in dynamic, consecutively, in order to track the evolution of scars.

This paper aims to achieve a parallelism between literature studies on imaging diagnosis, the characteristics of uterine scar and its evolution during pregnancy, and the obstetric consequences that can lead to increased maternal morbidity.

There has been a review of literature published between 2009-2017 on the international PubMed platform, which aimed at identifying the methods of diagnosis of cesarean post-cesarean scar, and the main changes in its characteristics during pregnancy, as well as the risks taken by the pregnant woman during a labor test for vaginal delivery after cesarean surgery. Gynecologic pathologies associated with post-caesarean scar have also been identified, but are not the subject of the current review.

\section{Discussions}

The first visualization of a post-cesarean scar was performed using hysterosalpingography in 1961. The ultrasound techniques followed, initially transabdominal in 1982, then transvaginal 1990 (3). At present, uterine scar and defects at this level can be evaluated by hysteroscopy, sonohysterography with contrast substance, gel-instilled sonohysterography, 3D transvaginal ultrasonography, even MRI.

There are no standardized criteria for choosing the time and technique evaluation of uterine scar after cesarean surgery. Most authors report the use of transabdominal or transvaginal ultrasound in the third trimester of pregnancy to measure and characterize the scar area at the level of the lower uterine segment, with three layers being highlighted by: amniotic membrane, myometrium and bladder wall $(4,5)$.

The imaging of uterine scar reveals two characteristic areas: the hypoechogenic area, called by some authors "niche" or "defect", and the scar area of the myometrium, called the "residual myometrium thickness" (distance from the endometrial defect to the uterine serosa). In most specialized studies, the characteristics of the hypoechogenic area are similar. Three measurements are made in the sagittal section of the uterus (the width of the niche, the depth of the niche and the thickness of the residual myometrium) and a cross-sectional measurement (length) $(2,4,5)$. Recent studies aim to highlight the changes in the dynamics that occur in the uterine scar, from 6 months after cesarean surgery, in the first, second and to the third trimester of pregnancy respectively.

Osser et al. proposed a classification of the size of uterine scar by calculating the percentage of the thickness of the residual myometrium from the thickness of the adjacent myometrium, and the increasing of this percentage along with the increased number of cesarean operations (6).

The importance of assessing the appearance and size of uterine scarring and its areas is due to the large number of complications that may occur in the pregnant woman with cesarean birth history: uterine rupture, dehiscence of the hysterorrhaphy, the pregnancy implantation in the uterine scar, placental anomalies (placenta previa and accreta/ percreta).

The quarterly assessment of uterine scar in pregnancy revealed an average increase of $1.8 \mathrm{~mm}$ in width per quarter, a decrease in depth and length averaging from $1.8 \mathrm{~mm}$ to $1.9 \mathrm{~mm}$ per quarter respectively, and a decrease in the thickness of the residual myometrium approximately $1.1 \mathrm{~mm}$ per quarter.

Naji et al. shows two cases of uterine rupture at which the thickness of the residual myometrium was measured and observed a decrease of $2.7 \mathrm{~mm}$ and 2.5 $\mathrm{mm}$ respectively in the second trimester versus the first trimester (2). Other studies in the literature reported a higher incidence of rupture uterine, when the lower uterine segment thickness fell below $2.3 \mathrm{~mm}$. A study of 62 pregnant women in the third trimester showed an average thickness of the $3.2 \mathrm{~mm}$ lower segment, with a higher thickness among multiparous women (7). 
Jastrow et al., by ultrasound measuring of uterine scar in the third trimester of pregnancy, has set a value of 1.4-2 $\mathrm{mm}$ in thickness in predicting complications like uterine rupture (8). Bujold et al., on a study of 263 pregnant women, aged between 35-38 weeks of gestation, regarding the rate of uterine rupture according to the thickness of the anterior uterine wall and the inferior segment (measured by transvaginal ultrasound), revealed an increase by more than $10 \%$ when the lower segment measured was below 2,5 mm (9).

The success of the labor test for vaginal birth after cesarean surgery is a recent subject, many authors trying to create protocols on predicting success in relation to the features of uterine scar. The failure of the labor test is associated with increased maternal and fetal morbidity. To date, however, there is no obvious evidence to associate the imaging aspect of postcesarean uterine scar and its function. Naji et al. conducted in 2013 a study on 121 pregnant women, which aimed to predict the success of the labor trial using the characteristics of uterine scar, assessed by transvaginal ultrasonography. The labor test was successful in $61 \%$ of cases, with the immediate result of giving birth by vaginal delivery. They concluded that the decrease in the thickness of the remaining myometrium seems to predict the success and not the hypoechogene area ("niche"), and the smaller it is in the second quarter versus the first quarter, the lower are the chances of success. The mean between the two quarters was lower in pregnant women that gave birth spontaneously $(0.8$ $\mathrm{mm}$ vs. $2.8 \mathrm{~mm})$. The same authors have made a mathematical computation formula for estimating the chances of vaginal delivery after cesarean surgery (10).

Another study, conducted in 2015 on a group of 142 pregnant women with a previous cesarean section, that followed the labor trial, showed a $67.6 \%$ success rate on vaginal birth. The thickness of uterine scar, measured in the second trimester (between 24-28 weeks of gestation), was lower in cases that resulted in cesarean delivery (11). Rozenberg et al. also found that the thickness of the lower uterine segment was lower in pregnant women who gave birth via elective cesarean, compared to those in whom labor tests were successful (3.8 mm vs. $4.5 \mathrm{~mm})(12)$.

An important element that has been the subject of studies in the literature over the past years is represented by the hysterorrhaphy technique in the cesarean surgery and the impact on postoperative uterine scar formation. Some authors consider, in relation to the risk of uterine rupture, that the suture in the monolayer interrupted suture poses a higher risk than the uninterrupted monolayer suture, which in turn presents an increased risk than double-layer hysterorrhaphy (13).

A study conducted in the year 2013 on 149 pregnant women (68 of them had mono-layer and 81 had doublelayer hysterorrhaphy) showed a larger average of the length of the "niche" in the monolayer suture group, but no difference in depth and width of the defect. The thickness of the myometrium in double-suture cases was significantly higher (by $1.2 \mathrm{~mm}$ ) (14). By comparison, Di Spiezio et al. in 2017, performing a meta-analysis of 9 randomized control studies on 3969 cases, regarding the hysterorrhaphy, showed the same incidence of defects at uterine scar level, dehiscence, and uterine rupture, regardless of the uterine suture technique used. The thickness of the remaining myometrium was also higher in double-suture cases (1).

One cause of the reduced size of the residual myometrium in the case of the monolayer interrupted suture can be represented by the poor vascularization obtained by excessive tissue trapping and consequently insufficient healing (14).

Uterine rupture is a complication with maternal and fetal morbidity increased with an incidence up to $0.45 \%$ for patients with a previous cesarean section. However, the rarity of such complications makes it difficult to establish a standardized technique for hysterorrhaphy, and further studies on a much larger number being needed for this purpose (1).

Another consequence, with possible complications leading to increased maternal morbidity, is represented by the pregnancy inserted in the uterine scar postcesarean surgery. The incidence is small, between 1: 1800-1: 2200 pregnancies (15). Diagnosis can be established by transvaginal ultrasound revealing an empty uterine cavity, closed cervical canal, gestational sac (triangular form below 8 weeks of gestation) with the vitelline vesicle and the embryo present, and the trophoblast at the scar level, the myometrium with the 1-3 mm thickness between the gestational sac and urinary bladder, and an increased vascular area in the uterine scar in the presence of a positive pregnancy test.

According to some authors, about $50 \%$ of the pregnancies implanted at the uterine scar level will evolve to spontaneous abortion during the first trimester of pregnancy (16). In the literature it was reported that the management in these cases differs. There is a medical one, a surgical treatment, but also expectant management. The latter has been shown to led to increased maternal morbidity, as most pregnancies 
evolving until the second trimester will evolve with placental complications like placenta accreta, percreta, etc. If pregnancies evolve up to near-term and end with the birth of living newborns, maternal complications such as bleeding, shock, uterine rupture ultimately lead to hysterectomy of necessity in almost all the cases (17). In a study conducted by Ballas et al., on 10 cases of pregnancy implanted at the uterine scar level, all pregnancies led to the birth of a living newborn, but they all needed a hysterectomy of necessity (18). Another similar study revealed in all cases placenta percreta from the histopathological examination performed on the hysterectomy sample (17).

\section{Conclusions}

As the incidence of caesarean birth has increased in recent years, interest in assessing the quality of uterine scar has also increased. The success of the labor test for vaginal delivery after cesarean surgery is difficult to predict, but it seems that the characteristics of uterine scar have an important role. New studies on a large group of patients, uniformity of criteria for the definition of uterine scar defects, hysterorrhaphy techniques are needed in order to standardize the relationship between uterine scar and birth prognosis in pregnant women with scar tissue after cesarean surgery. Complications that may occur in the presence of uterine scar lead to increased maternal and fetal morbidity, making it an important topic to be debate.

\section{Conflict of interest disclosure}

There are no known conflicts of interest in the publication of this article, and there was no financial support that could have influenced the outcomes. The manuscript was read and approved by all authors.

\section{References}

1. Di Spiezio Sardo A, Saccone G, McCurdy R, Bujold E, Bifulco G, Berghella V. Risk of Cesarean scar defect following single- vs double-layer uterine closure: systematic review and meta-analysis of randomized controlled trials. Ultrasound Obstet Gynecol. 2017; 50(5): 578-83. PMID: 28070914, DOI: 10.1002/uog.17401

2. Naji O, Daemen A, Smith A, et al. Changes in Cesarean section scar dimensions during pregnancy: a prospective longitudinal study. Ultrasound Obstet Gynecol. 2013; 41(5): 556-62. PMID: 23108803, DOI: 10.1002/uog.12334
3. Naji O, Daemen A, Smith A, et al. Visibility and measurement of Cesarean section scars in pregnancy: a reproducibility study. Ultrasound Obstet Gynecol. 2012; 40(5): 549-56. PMID: 22323065, DOI: 10.1002/uog.11132

4. Au H-K, Liu C-F, Tzeng C-R, Chien L-W. Association between ultrasonographic parameters of Cesarean scar defect and outcome of early termination of pregnancy. Ultrasound Obstet Gynecol. 2016; 47(4): 506-10. PMID: 25865778, DOI: $10.1002 /$ uog. 14877

5. Naji O, Abdallah Y, Bij De Vaate AJ, et al. Standardized approach for imaging and measuring Cesarean section scars using ultrasonography. Ultrasound Obstet Gynecol. 2012; 39(3): 252-59. PMID: 21858885, DOI: 10.1002/uog. 10077

6. Vikhareva Osser O, Jokubkiene L, Valentin L. High prevalence of defects in Cesarean section scars at transvaginal ultrasound examination. Ultrasound Obstet Gynecol. 2009; 34(1): 90-7. PMID: 19499514, DOI: 10.1002/uog.6395

7. Nassie D, Aviram A, Efrat Z, Meizner I, Wiznitzer A, Bardin R. P04.10: Intrapartum measurement of the lower uterine segment thickness (LUST): defining the normal. Ultrasound Obstet Gynecol. 2017; 50: 165-165. DOI: 10.1002/uog.18034

8. Jastrow N, Chaillet N, Roberge S, Morency A-M, Lacasse Y, Bujold E. Sonographic Lower Uterine Segment Thickness and Risk of Uterine Scar Defect: A Systematic Review. J Obstet Gynaecol Can. 2010; 32(4): 321-7. PMID: 20500938, DOI: 10.1016/S1701-2163(16)34475-9

9. Bujold E, Jastrow N, Simoneau J, Brunet S, Gauthier RJ. Prediction of complete uterine rupture by sonographic evaluation of the lower uterine segment. Am J Obstet Gynecol. 2009; 201(3): 320.e1-320.e6. PMID: 19733288, DOI: 10.1016/j.ajog.2009.06.014

10. Naji O, Wynants L, Smith A, et al. Predicting successful vaginal birth after Cesarean section using a model based on Cesarean scar features examined by transvaginal sonography. Ultrasound Obstet Gynecol. 2013; 41(6): 672-8. PMID: 23371440, DOI: 10.1002/uog.12423

11. Singh N, Tripathi R, Mala YM, Dixit R. Scar thickness measurement by transvaginal sonography in late second trimester and third trimester in pregnant patients with previous cesarean section: does sequential change in scar thickness with gestational age correlate with mode of delivery? $J$ 
Ultrasound. 2015; 18(2): 173-8. PMID: 26191105, DOI: $10.1007 / \mathrm{s} 40477-014-0116-3$

12. Rozenberg P, Goffinet F, Philippe HJ, Nisand Thickness of the lower uterine segment: its influence in the management of patients with previous cesarean sections. Eur J Obstet Gynecol Reprod Biol. 1999; 87(1): 39-45. PMID: 10579615

13. Roberge S, Chaillet N, Boutin A, et al. Singleversus double-layer closure of the hysterotomy incision during cesarean delivery and risk of uterine rupture. Int J Gynecol Obstet. 2011; 115(1): 5-10. PMID: 21794864, DOI: 10.1016/j.ijgo.2011.04.013

14. Huirne JAF, Stegwee SI, van der Voet LF, de Groot CJM, Hehenkamp WJK, Brölmann HAM. Re: Risk of Cesarean scar defect following single- vs doublelayer uterine closure. Ultrasound Obstet Gynecol. 2017; 50(5): 664-6. PMID: 29105218, DOI: 10.1002/uog.18901

15. Jurkovic D, Knez J, Appiah A, Farahani L, Mavrelos D, Ross JA. Surgical treatment of Cesarean scar ectopic pregnancy: efficacy and safety of ultrasound-guided suction curettage.
Ultrasound Obstet Gynecol. 2016; 47(4): 511-17. PMID: 26764166, DOI: 10.1002/uog.15857

16. Jurkovic D, Hillaby K, Woelfer B, Lawrence A, Salim R, Elson CJ. First-trimester diagnosis and management of pregnancies implanted into the lower uterine segment Cesarean section scar. Ultrasound Obstet Gynecol. 2003; 21(3): 220-27. PMID: 12666214, DOI: 10.1002/uog.56

17. Timor-Tritsch IE, Monteagudo A, Cali G, et al. Cesarean scar pregnancy is a precursor of morbidly adherent placenta. Ultrasound Obstet Gynecol. 2014; 44(3): 346-53. PMID: 24890256, DOI: 10.1002/uog.13426

18. Ballas J, Pretorius D, Hull AD, Resnik R, Ramos GA. Identifying sonographic markers for placenta accreta in the first trimester. J Ultrasound Med. 2012; 31(11): 1835-41. PMID: 23091257 